\title{
Evidence of nonadiabatic exciton-phonon interaction probed by second-order LO-phonon replicas of single quantum dots
}

Daniel Dufåker, Karlsson Fredrik, L O. Mereni, V Dimastrodonato, G Juska, E Pelucchi and

Per-Olof Holtz

\section{Linköping University Post Print}

\section{Tweet}

N.B.: When citing this work, cite the original article.

Original Publication:

Daniel Dufåker, Karlsson Fredrik, L O. Mereni, V Dimastrodonato, G Juska, E Pelucchi and Per-Olof Holtz, Evidence of nonadiabatic exciton-phonon interaction probed by second-order LO-phonon replicas of single quantum dots, 2013, Physical Review B. Condensed Matter and Materials Physics, (87), 8. http://dx.doi.org/10.1103/PhysRevB.87.085317

Licencee: American Physical Society

http://www.aps.org/

Postprint available at: Linköping University Electronic Press

http://urn.kb.se/resolve?urn=urn:nbn:se:liu:diva-90677 


\title{
Evidence of nonadiabatic exciton-phonon interaction probed by second-order LO-phonon replicas of single quantum dots
}

\author{
D. Dufåker, ${ }^{1}$ K. F. Karlsson, ${ }^{1}$ L. O. Mereni, ${ }^{2}$ V. Dimastrodonato, ${ }^{2}$ G. Juska, ${ }^{2}$ E. Pelucchi, ${ }^{2}$ and P. O. Holtz ${ }^{1}$ \\ ${ }^{1}$ Linköping University, Department of Physics, Chemistry, and Biology (IFM), Semiconductor Materials, S-58183 Linköping, Sweden \\ ${ }^{2}$ Tyndall National Institute, University College Cork, Ireland
}

(Received 14 December 2012; published 25 February 2013)

\begin{abstract}
In this experimental study of single InGaAs/GaAs quantum dots (QDs) the photoluminescence intensity of the second order LO-phonon replica of the excitonic interband recombination was measured along with the intensities of the first and zeroth orders. The results show that the intensity of the second-order replica is three to four times stronger than expected from the adiabatic Huang-Rhys theory, indicating that the neglected nonadiabaticity plays an important role for the understanding of the exciton-phonon coupling in QDs.
\end{abstract}

DOI: 10.1103/PhysRevB.87.085317

PACS number(s): 78.67.Hc, 78.55.Cr, 63.22.-m

A semiconductor quantum dot (QD) exhibits discrete energy levels in the same manner as atoms. Therefore, they are often referred to as artificial atoms. ${ }^{1}$ The QD acts as a trap for the charge carriers, i.e., electrons $(e)$ and holes $(h)$, that are excited in the semiconductor lattice. The trapped and quantum confined electrons and holes interact and form different kinds of exciton complexes, depending on the number of interacting charge carriers within the QD. Following optical recombination of these excitonic complexes there is a minor probability that some of the energy remains in the lattice in the form of a single or multiple quantized lattice vibrations, e.g., as longitudinal optical (LO) phonons. Whenever this occurs the photon energy is reduced by a corresponding multiple of the LO-phonon energy $\hbar \omega_{L O}$. This effect is experimentally revealed by the presence of so-called phonon replicas in the optical recombination spectra positioned an integral number of $\hbar \omega_{L O}$ below the zero-phonon emission.

The relative strength of the phonon replicas is commonly described within the Huang-Rhys formalism, for which the intensity of the $n$ th-order replica follows the Poisson distribution $I_{n}=I_{0} \cdot e^{-S} S^{n} / n$ !. Experimentally, the Huang-Rhys parameter $S$ can be determined as the ratio between the intensities of the first and zeroth order replicas. ${ }^{2}$ The HuangRhys parameter is, for the neutral exciton $(X=1 e+1 h)$, directly related to the exciton-phonon coupling strength.

Previous studies of the LO-phonon coupling related to single QDs have mainly been focused on the neutral exciton and biexciton $(2 X=2 e+2 h),{ }^{3-5}$ but the effect of additional charge has also been theoretically examined for GaAs microcrystallites ${ }^{6}$ and recently experimentally investigated for single InGaAs/AlGaAs QDs. ${ }^{7}$ Surprisingly, the Huang-Rhys parameter was found to be significantly reduced for the positively charged exciton $\left(X^{+}=1 e+2 h\right)$, as compared to $X$ and the negatively charged exciton $\left(X^{-}=2 e+1 h\right)$, and it has further been concluded that the exciton complexes couple to LO phonons within the QDs rather than in the barriers or in the neighboring vertical quantum wire. ${ }^{8,9}$

It has been suggested that nonadiabatic effects caused by phonon mediated scattering between excitonic states, neglected in the adiabatic Huang-Rhys approach, might play a dominant role in the description of the interaction between the confined charge carriers and LO phonons. ${ }^{7,10}$ For model computations of InAs/GaAs QDs, the nonadiabatic effects have been predicted to yield radical enhancements of the second-order phonon replica by about two orders of magnitude, as compared to the Huang-Rhys approach. ${ }^{7}$ Thus, access to the second-order phonon replica is required in order to experimentally test the actual significance of nonadiabatic effects in real QDs.

Experimental studies of the second-order phonon replica of QDs have been very scarce; a weak signature has been reported for ensembles of InAs/GaAs QDs. ${ }^{3}$ For single CdSe/ZnCdSe QDs, for which the polar coupling is stronger as compared to III-V QDs, the second-order LO-phonon replica has been detected. However, in this case, the intensity was not extracted and the validity of the Huang-Rhys theory could accordingly not be evaluated. ${ }^{5}$

In this paper, we present experimental results of excitonic LO-phonon replicas up to the second order for single $\mathrm{In}_{0.25} \mathrm{Ga}_{0.75} \mathrm{As} / \mathrm{GaAs}$ QDs. The pyramidal QD system chosen for this study is of particular technological interest: These site-controlled dots can exhibit high spectral purity with very high dot-to-dot uniformity, ${ }^{11,12}$ while the system is fully compatible with slab photonic crystal technology. ${ }^{13}$ The predicted threefold symmetry of these dots ${ }^{14}$ was recently confirmed experimentally ${ }^{15}$ and enabled the realization of site-controlled sources of polarization entangled photons. ${ }^{16}$ For such applications, dephasing is a critical issue, and the LOphonon mediated interaction of QD states with the surrounding matrix can provide significant contribution to dephasing. ${ }^{17}$

The sample used, in this study, was grown by low-pressure metal organic vapor phase epitaxy on a prepatterned GaAs (111)B substrate, in which inverted tetrahedral micropyramids make up the 7.5- $\mu \mathrm{m}$ pitched pattern. Particular care is taken to monitor unintentional impurity levels during the growth. ${ }^{17-19}$ QDs are self-formed at the inverted pyramids during deposition of the $\operatorname{In}_{0.25} \mathrm{Ga}_{0.75}$ As QD layer (nominally $0.5 \mathrm{~nm}$ thick) due to capillarity effects and decomposition rate anisotropy. ${ }^{20,21}$ The QD layer is sandwiched between two GaAs barrier layers (see inset of Fig. 1). In order to increase light extraction efficiency, the samples underwent a substrate-removal procedure called backetching. ${ }^{22,23}$ It should be emphasized that the signal of the second-order phonon replica is too weak to be detected without backetching.

The QDs were kept at a temperature of $4 \mathrm{~K}$ in the microphotoluminescence ( $\mu \mathrm{PL}$ ) setup and excited individually, 
using a Ti-sapphire laser, with a wavelength of $732 \mathrm{~nm}$ and a power density of about $100 \mathrm{nW} / \mu \mathrm{m}^{2}$ focused to a spot of about $2 \mu \mathrm{m}$ in diameter using a microscope objective with a numerical aperture of 0.55 . A single grating monochromator (1200 grooves $/ \mathrm{mm}$ blazed for $750 \mathrm{~nm}$, focal length $0.55 \mathrm{~m}$ ) with a spectral resolution of $\sim 0.1 \mathrm{meV}$ equipped with a CCD camera was used to acquire spectra of single QDs. In order to improve the data quality at simultaneous detection of the zeroth- and the first-order replicas (the first- and the second-order replicas) the stronger replica of lower order was suppressed before detection. In this way, saturation of the CCD by the strongest emission lines could be avoided for long integration times. The intensity ratio between two consecutive replicas is for these QDs in the range 100-1000, and a neutral density filter transmitting $1.46 \%$ was placed directly in front of one-half of the CCD chip to accomplish the desired suppression of the high-intensity emission. Typical integration times used are $\sim 10 \mathrm{~s}$ for most of the measurements and $120 \mathrm{~s}$ for those involving the second-order replicas.

The average number of electrons and holes populating the QD is determined by the excitation power. These $\mathrm{In}_{0.25} \mathrm{Ga}_{0.75} \mathrm{As} / \mathrm{GaAs}$ QDs exhibit negative biexciton binding energies, and power dependence in combination with time-resolved PL measurements has been performed for the identification of the exciton and the biexciton. As expected, the biexciton exhibits a quadratic power dependence and faster recombination time than the exciton. ${ }^{24,25}$

The Huang-Rhys parameter and the full width at half maximum (FWHM) of the emission lines were determined from several spectra, where the zero-phonon emission and the first-order LO-phonon replica were measured simultaneously. The Huang-Rhys parameter is determined as the integrated intensity ratio between first- and zeroth-order replica. Average values from eleven different QDs are displayed in Fig. 1,

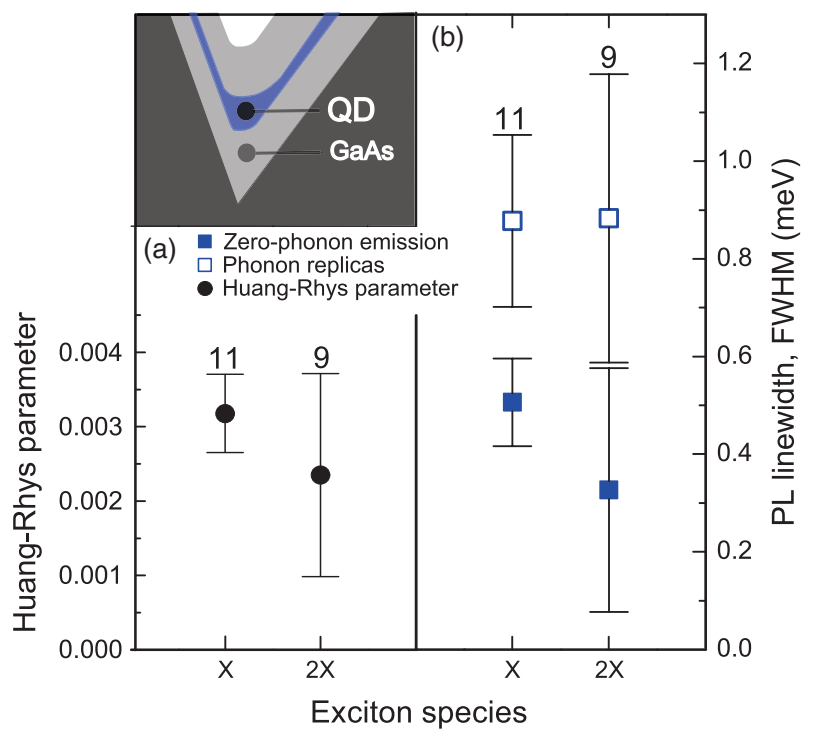

FIG. 1. (Color online) (a) The measured Huang-Rhys parameter (b) and linewidths (FWHM) represented by the mean values of in total eleven different $\operatorname{In}_{0.25} \mathrm{Ga}_{0.75} \mathrm{As} / \mathrm{GaAs}$ QDs. The bars indicate the standard deviation from the mean, and the numbers above indicate the number of measured QDs. The inset shows a schematic twodimensional side view of the pyramidal QDs used in the study.

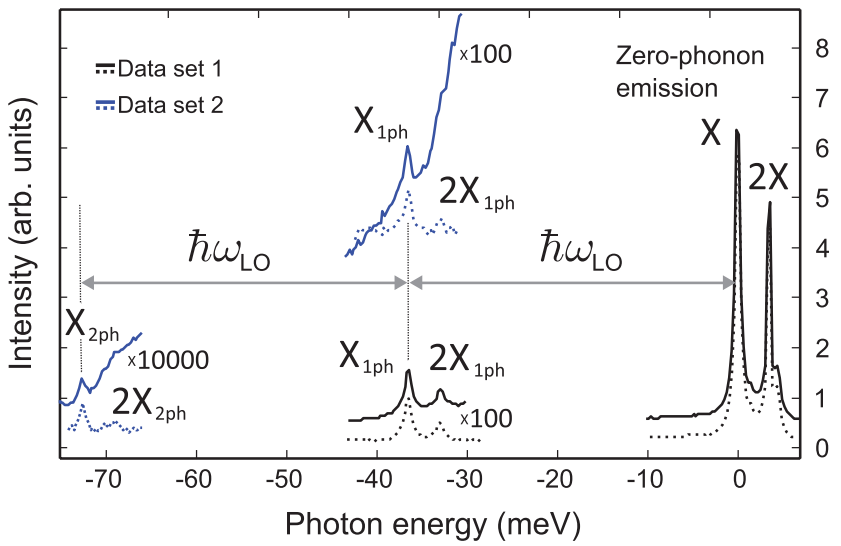

FIG. 2. (Color online) $\mu \mathrm{PL}$ data of a single QD. Data set 1 (black) is spectra of the zero-phonon emission and the corresponding first order LO-phonon emission (enhanced 100 times) for one $\mathrm{In}_{0.25} \mathrm{Ga}_{0.75} \mathrm{As} / \mathrm{GaAs}$ QD. Data set 2 (blue) consists of an average of 28 spectra, each measuring the first- and second-order LO-phonon replicas simultaneously (second-order emission is enhanced 100 times with respect to first-order emission). For both data sets, the dotted lines show spectra with the background removed. The energy of the zero-phonon emission for the neutral exciton $X$ (at about $1443 \mathrm{meV}$ ) is set to zero.

demonstrating a Huang-Rhys parameter for $X$ of about $S \approx$ 0.003 and a FWHM linewidth of the first-order replica about twice the width of the corresponding zero-phonon emission. Also, the values corresponding to the biexciton $2 X$ are shown for completeness. The corresponding spectra for a single QD with $S=0.0033( \pm 0.0005)$ are shown in Fig. 2 (black curves). By averaging 28 individual long-exposure spectra from a selected QD, also the second-order phonon replica could be clearly resolved (see blue curves of Fig. 2), with a relative integrated intensity of about $2.0 \times 10^{-5}\left( \pm 0.8 \times 10^{-5}\right)$ with respect to that of the zero-phonon emission. All intensities were integrated after background removal (see Fig. 2).

The measured intensity of the second-order replica is three to four times stronger than what is expected from the Huang-Rhys theory for $S=0.0033$. This significant discrepancy is attributed to nonadiabatic effects neglected in the Huang-Rhys theory. The effect of nonadiabacity on the exciton-phonon interaction has been investigated theoretically by Verzelen et al. in Ref. 7, where numerical modeling of InAs/GaAs QDs predicted the intensity of the second-order phonon replica to be two orders of magnitude stronger than what is achieved with Huang-Rhys theory. ${ }^{7}$ Such a dramatic significance of nonadiabatic effects is obviously not observed in the experimental data of this work. The exact reason for this discrepancy remains unclear, since the electronic structure of the measured dot is not expected to be radically different from the dots modeled in Ref. 7.

The LO-phonon energy for the neutral exciton $X$ of the QD presented in Fig. 2 is $\hbar \omega_{L O}=36.5 \mathrm{meV}$. It is worth mentioning that the data represented by the blue curves in Fig. 2 exhibit minor peaks at energy distances $\hbar \omega_{L O}$ and $2 \hbar \omega_{L O}$, respectively, from the $2 X$ zero-phonon emission. These weaker peaks are interpreted as the first- and secondorder phonon replicas of the biexciton. The spectral linewidths of $X$ for the zeroth-, first-, and second-order emission are 


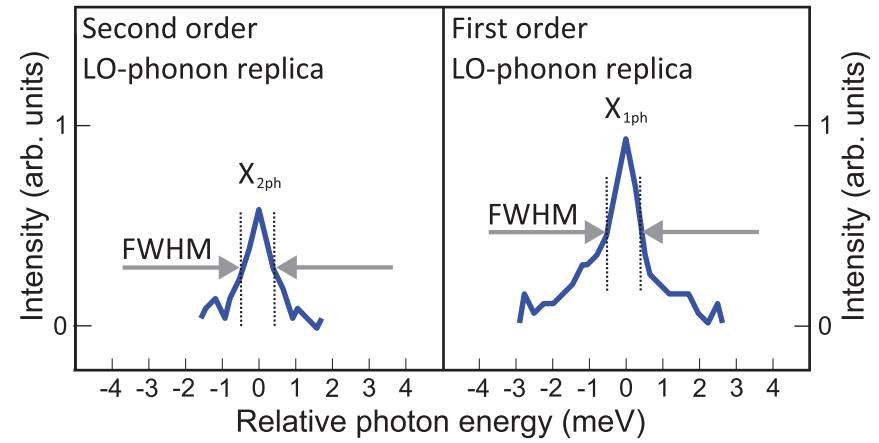

FIG. 3. (Color online) Enlargement of data in Fig. 2 (background removed). The FWHM is marked with arrows in the figure. The distances between the arrow heads in both panels are equal.

$0.6( \pm 0.1), 0.8( \pm 0.2)$, and $0.8( \pm 0.2) \mathrm{meV}$, respectively (see Fig. 3). The increase of the linewidth by $0.2 \mathrm{meV}$ for the first-order phonon emission replica has previously been attributed to variations of the In composition as well as the strain within the QDs, causing a broadened distribution of the phonon modes. ${ }^{9}$ Note that the linewidth of the second-order phonon replica here remains equal to the first-order replica (see Fig. 3), which is surprising since a distribution of phonon modes should contribute to an additional broadening of the second-order replica.

In conclusion, we have performed an experiment allowing us to unambiguously resolve the second order LO-phonon replica for a single InGaAs/GaAs QD, exhibiting a PL intensity five orders of magnitude weaker than the main QD emission. With spectral access to both the first- and the second-order phonon replicas, the validity of the Huang-Rhys theory for QDs could be tested. It was found that the second phonon replica is three to four times stronger than expected from the Huang-Rhys theory, and this significant discrepancy can be attributed to nonadiabatic effects neglected by this theory. However, the predicted strong modification $\left(\sim 10^{2}\right)$ of the intensity of the second-order phonon replica due to adiabatic effects is not supported by our experiments. ${ }^{710}$ Instead, the data suggest that nonadiabatic effects are not as significant as expected from the current theoretical models of the phonon-exciton interaction and calls for a deeper theoretical understanding than the one currently available.

This research was enabled by the Irish Higher Education Authority Program for Research in Third Level Institutions (2007-2011) via the INSPIRE program, and by Science Foundation Ireland under Grants No. 05/IN.1/I25 and No. 10/IN.1/I3000 and by grants from the Swedish Research Council (VR) and by equipment grants from the K. A. Wallenberg Foundation. We also acknowledge support from the Swedish Government Strategic Research Area in Materials Science on Functional Materials at Linköping University (Faculty Grant SFO-Mat-LiU No. 2009-00971). We are grateful to Dr. $\mathrm{K}$. Thomas for his support with the MOVPE system.
${ }^{1}$ D. Gammon, Nature (London) 405, 899 (2000).

${ }^{2}$ K. Huang and A. Rhys, Proc. R. Soc. London A 204, 406 (1950).

${ }^{3}$ R. Heitz, I. Mukhametzhanov, O. Stier, A. Madhukar, and D. Bimberg, Phys. Rev. Lett. 83, 4654 (1999).

${ }^{4}$ P. Hawrylak, G. A. Narvaez, M. Bayer, and A. Forchel, Phys. Rev. Lett. 85, 389 (2000).

${ }^{5}$ F. Gindele, K. Hild, W. Langbein, and U. Woggon, Phys. Rev. B 60, R2157 (1999).

${ }^{6}$ S. Nomura and T. Kobayashi, Phys. Rev. B 45, 1305 (1992).

${ }^{7}$ O. Verzelen, R. Ferreira, and G. Bastard, Phys. Rev. Lett. 88, 146803 (2002).

${ }^{8}$ D. Dufåker, K. F. Karlsson, V. Dimastrodonato, L. O. Mereni, B. E. Sernelius, P. O. Holtz, and E. Pelucchi, Phys. Rev. B 82, 205421 (2010).

${ }^{9}$ D. Dufåker, L. O. Mereni, K. F. Karlsson, V. Dimastrodonato, G. Juska, P. O. Holtz, and E. Pelucchi, Appl. Phys. Lett. 98, 251911 (2011).

${ }^{10}$ V. M. Fomin, V. N. Gladilin, J. T. Devreese, E. P. Pokatilov, S. N. Balaban, and S. N. Klimin, Phys. Rev. B 57, 2415 (1998).

${ }^{11}$ K. Leifer, E. Pelucchi, S. Watanabe, F. Michelini, B. Dwir, and E. Kapon, Appl. Phys. Lett. 91, 081106 (2007).

${ }^{12}$ L. O. Mereni, V. Dimastrodonato, R. J. Young, and E. Pelucchi, Appl. Phys. Lett. 94, 223121 (2009).

${ }^{13}$ P. Gallo, M. Felici, B. Dwir, K. A. Atlasov, K. F. Karlsson, A. Rudra, A. Mohan, G. Biasiol, L. Sorba, and E. Kapon, Appl. Phys. Lett. 92, 263101 (2008).
${ }^{14}$ K. F. Karlsson, M. A. Dupertuis, D. Y. Oberli, E. Pelucchi, A. Rudra, P. O. Holtz, and E. Kapon, Phys. Rev. B 81, 161307 (2010).

${ }^{15}$ M. A. Dupertuis, K. F. Karlsson, D. Y. Oberli, E. Pelucchi, A. Rudra, P. O. Holtz, and E. Kapon, Phys. Rev. Lett. 107, 127403 (2011).

${ }^{16}$ G. Juska, V. Dimastrodonato, L. O. Mereni, A. Gocalinska, and E. Pelucchi (unpublished).

${ }^{17}$ A. Carmele, F. Milde, M.-R. Dachner, M. B. Harouni, R. Roknizadeh, M. Richter, and A. Knorr, Phys. Rev. B 81, 195319 (2010).

${ }^{18}$ E. Pelucchi, N. Moret, B. Dwir, D. Y. Oberli, A. Rudra, N. Gogneau, A. Kumar, E. Kapon, E. Levy, and A. Palevski, J. Appl. Phys. 99, 093515 (2006)

${ }^{19}$ V. Dimastrodonato, L. Mereni, R. Young, and E. Pelucchi, J. Cryst. Growth 312, 3057 (2010).

${ }^{20}$ E. Pelucchi, V. Dimastrodonato, A. Rudra, K. Leifer, E. Kapon, L. Bethke, P. A. Zestanakis, and D. D. Vvedensky, Phys. Rev. B 83, 205409 (2011)

${ }^{21}$ V. Dimastrodonato, E. Pelucchi, and D. D. Vvedensky, Phys. Rev. Lett. 108, 256102 (2012).

${ }^{22}$ A. Hartman, Y. Ducommun, K. Leifer, and E. Kapon, J. Phys.: Condens. Matter 11, 5901 (1999).

${ }^{23}$ V. Dimastrodonato, L. O. Mereni, R. J. Young, and E. Pelucchi, Phys. Status Solidi B 247, 1862 (2010).

${ }^{24}$ K. Brunner, G. Abstreiter, G. Böhm, G. Tränkle, and G. Weimann, Phys. Rev. Lett. 73, 1138 (1994).

${ }^{25}$ R. M. Thompson, R. M. Stevenson, A. J. Shields, I. Farrer, C. J. Lobo, D. A. Ritchie, M. L. Leadbeater, and M. Pepper, Phys. Rev. B 64, 201302 (2001). 